\title{
Ramsey's theorem for colors from a metric space
}

\author{
Mikołaj Bojańczyk • Eryk Kopczyński • \\ Szymon Toruńczyk
}

Received: 16 August 2010 / Accepted: 26 April 2012 / Published online: 11 May 2012

(C) The Author(s) 2012. This article is published with open access at Springerlink.com

\begin{abstract}
The Ramsey theorem says that for any countably infinite undirected clique whose edges are colored by a finite number of colors, there is an infinite subclique whose edges are colored by a single color. In this note, we generalize the theorem to a situation where the colors form a compact metric space.
\end{abstract}

Keywords Ramsey theorem · Metric compact space · Compact semigroups

By $[A]^{2}$ we denote the family of size two subsets of $A$, interpreted as edges in the undirected graph with nodes $A$. If $K$ is a metric space and $A$ is a set, we say that $k \in K$ is the limit of a function $f:[A]^{2} \rightarrow K$ if for any $\epsilon>0$, there is some finite subset $C \subseteq A$ such that the image of $f$, when restricted to $[A-C]^{2}$, is contained in the ball of radius $\epsilon$ and center $k$.

Theorem 1 Let $K$ be a metric compact space, $B$ a countably infinite set, and $f$ : $[B]^{2} \rightarrow K$. Then there exists $a k \in K$ and an infinite subset $A \subseteq B$ such that the restriction of $f$ to $[A]^{2}$ has limit $k$.

Ramsey's theorem is a special case of the above theorem, when $K$ is equipped with a discrete metric. The proof is a simple adaptation of the proof of Ramsey's theorem.

Communicated by Jean-Eric Pin.

M. Bojańczyk and S. Toruńczyk were supported by ERC Starting Grant "Sosna”. E. Kopczyński was supported by the Polish Ministry of Science Grant Nr. N N206 567840.

M. Bojańczyk $(\bowtie) \cdot$ E. Kopczyński · S. Toruńczyk

Institute of Informatics, University of Warsaw, ul. Banacha 2, 02-097 Warszawa, Poland

e-mail: bojan@mimuw.edu.pl 
Proof Without loss of generality we assume that $B=\omega$. By induction, we will construct a sequence of sequences $a^{0}, a^{1}, a^{2}, \ldots \in B^{\omega}$, such that $a^{n}$ is a subsequence of $a^{n-1}$. We write $a_{m}^{n}$ for the $m$-the element of the $n$-th sequence $a^{n}$.

Let $a_{m}^{0}=m$. For $n>0$, let $a^{n}$ be a subsequence of $a^{n-1}$ such that $a_{m}^{n}=a_{m}^{n-1}$ for $m \leq n$, and the sequence

$$
f\left(\left\{a_{n}^{n}, a_{n}^{n}\right\}\right), f\left(\left\{a_{n}^{n}, a_{n+1}^{n}\right\}\right), f\left(\left\{a_{n}^{n}, a_{n+2}^{n}\right\}\right), \ldots
$$

is convergent to some $k_{n}$. This subsequence exists because $K$ is compact. Moreover, we can choose the sequence $a^{n}$ so that for all $m>n$ the distance from $f\left(\left\{a_{n}^{n}, a_{m}^{n}\right\}\right)$ to $k_{n}$ is at most $1 / n$.

Let $\left(q_{n}\right)$ be a sequence of numbers such that $k_{q_{n}}$ is convergent to some $k$ and moreover, $\delta\left(k_{q_{n}}, k\right) \leq 1 / n$. Define $A$ as $\left\{a_{q_{n}}^{q_{n}}: n \in \omega\right\}$.

Let $n<m$. Since $a^{q_{m}}$ is a subsequence of $a^{q_{n}}$, we have that $a_{q_{m}}^{q_{m}}=a_{z}^{q_{n}}$ for some $z>q_{m}>q_{n}>n$.

$$
\begin{aligned}
\delta\left(f\left(\left\{a_{q_{n}}^{q_{n}}, a_{q_{m}}^{q_{m}}\right\}\right), k\right) & \leq \delta\left(f\left(\left\{a_{q_{n}}^{q_{n}}, a_{q_{m}}^{q_{m}}\right\}\right), k_{q_{n}}\right)+\delta\left(k_{q_{n}}, k\right) \\
& \leq \delta\left(f\left(\left\{a_{q_{n}}^{q_{n}}, a_{q_{m}}^{q_{m}}\right\}\right), k_{q_{n}}\right)+1 / n \\
& =\delta\left(f\left(\left\{a_{q_{n}}^{q_{n}}, a_{z}^{q_{n}}\right)\right\}, k_{q_{n}}\right)+1 / n \\
& \leq 1 / n+1 / n=2 / n
\end{aligned}
$$

Since $\delta\left(f\left(\left\{a_{q_{n}}^{q_{n}}, a_{q_{m}}^{q_{m}}\right\}\right), k\right)<2 / n$, the set $A$ satisfies our claim.

The theorem assumes that the space $K$ is metric. We show a space $K$ which is compact but not metric, and where the statement of the theorem fails. Let $K$ be any compact space where not every sequence has a convergent subsequence. For instance, $K$ can be an uncountable product of unit intervals. Take some sequence $a_{1}, a_{2}, \ldots$ in $K$ which does not have any convergent subsequence, and define $f:[\omega]^{2} \rightarrow K$ by $f(\{i, j\})=a_{i}$. The theorem, when applied to this function $f$, would imply that there is a converging subsequence of $a_{1}, a_{2}, \ldots$.

By induction on $k$, one can generalize the theorem to functions $f$ defined on $k$-element subsets.

In the following corollary, we see what happens when $K$ has a semigroup structure. If $x \in K^{\omega}$ is a sequence of elements from a semigroup $K$, we write $x[i . . j$ ) for the multiplication of $x_{i} \cdots x_{j-1}$.

Corollary 1 If in Theorem 1 , the space $K$ has a semigroup structure with continuous multiplication, $B=\omega$, and $f$ is obtained from a sequence $x \in K^{\omega}$ by setting $f(\{i, j\})=x[i . . j)$, then the limit $k$ is idempotent, i.e. $k=k \cdot k$.

Proof Apply Theorem 1, yielding a set $A=\left\{u_{1}<u_{2}<\ldots\right\} \subseteq \omega$. If the action is continuous, then $k$ is idempotent, since

$$
k \cdot k=\left.\left.\lim _{n \rightarrow \infty} x\right|_{u_{n+1}} ^{u_{n}} \cdot \lim _{n \rightarrow \infty} x\right|_{u_{n+2}} ^{u_{n+1}}=\left.\left.\lim _{n \rightarrow \infty} x\right|_{u_{n+1}} ^{u_{n}} \cdot x\right|_{u_{n+2}} ^{u_{n+1}}=\left.\lim _{n \rightarrow \infty} x\right|_{u_{n+2}} ^{u_{n}}=k .
$$

In some papers, e.g. [1], a compact semigroup is a semigroup $S$ with compact topology such that the mapping $t \mapsto s \cdot t$ is required to be continuous for each $s \in S$. 
This assumption is weaker than our assumption from Corollary 1 that the action in $S$ is continuous.

However, the weaker assumption is not sufficient for idempotence of $s$ in Corollary 1 . Indeed, consider the semigroup $S=\{0,1,2, \ldots, \omega, \omega+1\}$, with the action $a \oplus b=\min (a+b, \omega+1)$, and the distance $\delta(a, b)=|f(a)-f(b)|$, where $f(n)=1 /(n+1), f(\omega)=0, f(\omega+1)=-1$. This semigroup is a compact semigroup in the meaning from [1]. Now, let $x_{n}=n$. If we apply Corollary 1 to $x, s$ has to be $\omega$, but $\omega$ is not idempotent: $\omega \oplus \omega=\omega+1$.

Open Access This article is distributed under the terms of the Creative Commons Attribution License which permits any use, distribution, and reproduction in any medium, provided the original author(s) and the source are credited.

\section{References}

1. Furstenberg, H., Katznelson, Y.: Idempotents in compact semigroups and Ramsey theory. Isr. J. Math. 68(3), 257-270 (1989). doi:10.1007/BF02764984 